\title{
Anomalous width variation of rarefactive ion acoustic solitary waves in the context of auroral plasmas
}

\author{
S. S. Ghosh and G. S. Lakhina \\ Indian Institute of Geomagnetism, Kalamboli, New Panvel (W), Navi Mumbai, 410 218, India \\ Received: 22 September 2003 - Revised: 13 February 2004 - Accepted: 23 February 2004 - Published: 14 April 2004 \\ Part of Special Issue "International Workshops on Nonlinear Waves and Chaos in Space Plasmas"
}

\begin{abstract}
The presence of dynamic, large amplitude solitary waves in the auroral regions of space is well known. Since their velocities are of the order of the ion acoustic speed, they may well be considered as being generated from the nonlinear evolution of ion acoustic waves. However, they do not show the expected width-amplitude correlation for $\mathrm{K}-\mathrm{dV}$ solitons. Recent POLAR observations have actually revealed that the low altitude rarefactive ion acoustic solitary waves are associated with an increase in the width with increasing amplitude. This indicates that a weakly nonlinear theory is not appropriate to describe the solitary structures in the auroral regions. In the present work, a fully nonlinear analysis based on Sagdeev pseudopotential technique has been adopted for both parallel and oblique propagation of rarefactive solitary waves in a two electron temperature multiion plasma. The large amplitude solutions have consistently shown an increase in the width with increasing amplitude. The width-amplitude variation profile of obliquely propagating rarefactive solitary waves in a magnetized plasma have been compared with the recent POLAR observations. The width-amplitude variation pattern is found to fit well with the analytical results. It indicates that a fully nonlinear theory of ion acoustic solitary waves may well explain the observed anomalous width variations of large amplitude structures in the auroral region.
\end{abstract}

\section{Introduction}

Solitary waves moving parallel to the background magnetic field are found to be ubiquitous throughout the magnetosphere including the plasma sheet boundary (Matsumoto et al., 1994; Franz et al., 1998; Cattell et al., 1999), the bow shock (Bale et al., 1998), within the solar wind (Mangeney et al., 1999) and at high altitude cusp regions (Cattell et al.,

Correspondence to: S. S. Ghosh

(sukti@iigs.iigm.res.in)
1999). They are also observed in strong currents, such as those associated with auroral acceleration region (Temerin et al., 1982; Böstrom et al., 1988; Mozer et al., 1997; Ergun et al., 1998; Bounds et al., 1999), where magnetic field aligned electron and ion beams are being accelerated and form aurora as they penetrate into the earth's upper atmosphere (Böstrom et al., 1989; Mozer et al., 1997). Though many of them show positive potential structures moving with velocities up to $5000 \mathrm{~km} / \mathrm{s}$ (Ergun et al., 1998), solitary waves observed in the auroral region often have much slower speeds and exhibit negative polarity (Cattell et al., 2001). The first observations of solitary waves and weak double layers were reported by the S3-3 group (Temerin et al., 1982). Later, these observations were substantially improved by the measurements of the Viking satellites (Böstrom et al., 1988). A series of expeditions followed thereafter by GEOTAIL (Matsumoto et al., 1994), FREJA (Donver et al., 1994), POLAR (Mozer et al., 1997) and FAST (McFadden et al., 1999; Ergun et al., 1998) satellites. The interpretation of the observed solitary waves and weak double layer like structures pose a serious challenge for theoretical space plasma physics. They draw even more attention because of their significant roles in accelerating particles in the auroral region and in ionosphere-magnetosphere interactions (Mäikii et al., 1993; Koskinen et al., 1990). The first wave of theoretical analysis (Lotko, 1983; Lotko and Kennel, 1983; Lotko, 1986) and computer simulations (Barnes et al., 1985; Hudson et al., 1983; Chanteur et al., 1983) were initiated immediately after the S3-3 measurements. Later, the trend was continued by many others (Marchenko and Hudson, 1995; Muschietti et al., 1999a,b; Mäikii et al., 1993; Berthomier et al., 1998; Dubouloz et al., 1991). The observed solitary structures are broadly categorized into ion and electron modes respectively. The electron mode solitary waves move with much faster velocities compared to ion modes, being of the order of the electron drift velocity (Muschietti et al., 1999a). Fast moving pulses with positive polarities were best observed by FAST satellites in the downward current regions of the auroral zone. 
Similar structures have also been observed by POLAR satellite at higher altitudes (Cattell et al., 2001). These electron solitary waves were first identified by GEOTAIL satellite as the constituents of the broadband electrostatic noise (BEN) (Muschietti et al., 1999a) and were found to be associated with electron beams (Crumley et al., 2001). The theoretical interpretations of these electron solitary waves mostly describe them as BGK (Bernstein-Greene-Kruskal) electron phase space holes (Turikov, 1984; Muschietti et al., 1999a,b, 2002; Maslov and Schamel, 1993; Schamel, 1982) while Dubouloz et al. (1991) has described them as electron acoustic waves. Ion mode solitary waves, on the other hand, were associated with up-flowing ion beams (Bounds et al., 1999) and move with a velocity of the order of ion sound wave (Dombeck et al., 2001). They are observed comparatively at lower altitudes of auroral zone (Cattell et al., 2001). The structures were found to show density depletion in the form of a symmetric potential well and are referred to as solitary waves (SW) but frequently they also display a net potential drop constituting weak double layers (WDL) (Temerin et al., 1982; Böstrom et al., 1989). Initial interpretations of such solitary waves were based on the theory of nonlinear evolution of coherent potential pulses from the linear ion acoustic waves (Lotko, 1983). Later the work was extended to include $\mathrm{H}^{+}$and $\mathrm{O}^{+}$beams (Qian et al., 1989). The problem of the formation of weak double layers was also addressed on the basis of the theory of ion acoustic waves (Gray et al., 1991) and was suggested as the result of the evolution of ion acoustic solitary waves (Berthomier et al., 1998). The possible existence of solitary waves in the ion acoustic frequency range triggered several theoretical and numerical investigations (Qian et al., 1988; Koskinen et al., 1990; Gray et al., 1991; Yadav and Sharma, 1990; Reddy and Lakhina, 1991; Reddy et al., 1992; Cairns et al., 1995; Sato and Okuda, 1980). In most of the cases they were described as rarefactive ion acoustic solitary waves and their analysis were based on a weakly nonlinear theory such as the K-dV equation (Washimi and Taniuti, 1966; Nishihara and Tajiri , 1981; Buti, 1980a). However, serious discrepancies exist between the analytical prediction and the observed phenomena regarding their width-amplitude characteristics. According to the weakly nonlinear theory, the width of a solitary wave is expected to decrease monotonically with an increasing amplitude. The exploration of Viking satellite, on the other hand, revealed that no such correlation exists for the observed ion solitary waves (Böstrom et al., 1989). Mäikii and Koskinen (1989) and others (Mäikii et al., 1993; Schamel, 1982; Dupree, 1982) tried to explain it interpreting them as BGK ion phase space holes. The debate still remained whether any such solitary wave can be described on the basis of the evolution of ion acoustic mode (Berthomier et al., 1998). A small amplitude theory remains inadequate for the process while a complete theory of large amplitude solitary waves is yet awaited. A particular lacuna lies in the overall width-amplitude profiles of such large amplitude rarefactive structures. In spite of extensive analytical investigations for the last two decades, scant attention has been given so far to their width-amplitude characteristics. In our previous works (Ghosh et al., 1996; Ghosh and Iyengar, 1997), we have shown that for a large amplitude rarefactive ion acoustic solitary wave, the width indeed increases with the increasing amplitude contradicting the prediction of the weakly nonlinear theory whereas in the small amplitude limit, it follows the usual variation pattern for the K-dV soliton. Thus the overall width-amplitude variation profile of a rarefactive ion acoustic solitary wave shows two distinct regions, namely the "decreasing width" (region I) and "increasing width" (region III) (Ghosh et al., 1996; Ghosh and Iyengar, 1997) respectively. The behavior pattern was found to be very much consistent over the wide parameter regions for an unmagnetized plasma. At that time we suggested that a similar behavior might be observed in the auroral acceleration region where large amplitude ion pulses with negative polarities are found to exist by several satellite expeditions (Ghosh and Iyengar, 1997, 2002). Recent analysis of Dombeck et al. (2001) using data from POLAR observations have revealed that for a rarefactive ion mode (SW) the width actually tends to increase with the increasing amplitude. A similar trend has also been observed by Cattell et al. (2001) for electron solitary waves as well. This motivated us to extend our previous investigations to a magnetized plasma with beam ions relevant to the auroral acceleration region. A fully nonlinear analysis has been adopted for a fluid plasma assuming an oblique propagation with the ambient magnetic field. For a parallel or near parallel propagation, the plasma is assumed to be unmagnetized. The width variation profile of the analytical solution has been compared with the observations of Dombeck et al. (2001). It indicates that the observed width-amplitude variation patterns of the rarefactive ion mode in the auroral acceleration region are consistent with the analytical results obtained for a fully nonlinear rarefactive ion acoustic solitary wave theory.

The paper is organized as follows. In the next section (Sect. 2) fully nonlinear Sagdeev pseudopotentials have been derived analytically for both unmagnetized and magnetized plasmas. The width-amplitude variation profiles for the corresponding rarefactive ion acoustic solitary wave solutions have been studied in Sect. 3. The overall width-amplitude variation profiles have been compared with the observations of Dombeck et al. (2001). A critical discussion has been included in Sect. 4.

\section{Derivation of the Sagdeev pseudopotential}

In the present analysis, fully nonlinear solutions for rarefactive ion acoustic solitary waves have been obtained considering two different cases, viz, parallel or near-parallel propagations and oblique propagations. A Sagdeev pseudopotential technique has been adopted to get the required solutions (Ghosh et al., 1996). For both the cases, the plasma is assumed to have two electron species with different temperatures where both the species are separately in thermal equilibrium. The cool electrons forming the background 
population are assumed to be of ionospheric origin while the hotter ones are those originated from the heated electron population of the magnetosphere. The plasma is assumed to have warm multi-ion species and a fluid model has been considered throughout the analysis. The massive ions are assumed to be adiabatic $(\gamma=3)$ for both the cases (Ghosh et al., 1996; Roychoudhury and Bhattacharyya, 1987).

\subsection{Case I: Parallel propagation (unmagnetized plasma)}

The plasma is assumed to be infinite, one-dimensional and collision-less. It is assumed to have two singly charged ion species of different masses. Since the propagation of the wave is along (parallel to) the magnetic field, the plasma is assumed to be unmagnetized. We have started with the usual warm ion (adiabatic) fluid equations (Ghosh and Iyengar, 2002)

$$
\begin{aligned}
& \frac{\partial n_{\mathrm{ih}}}{\partial t}+\frac{\partial\left(n_{\mathrm{ih}} v_{\mathrm{ih}}\right)}{\partial x}=0, \\
& \frac{\partial n_{\mathrm{il}}}{\partial t}+\frac{\partial\left(n_{\mathrm{il}} v_{\mathrm{il}}\right)}{\partial x}=0, \\
& \frac{\partial v_{\mathrm{ih}}}{\partial t}+v_{\mathrm{ih}} \frac{\partial v_{\mathrm{ih}}}{\partial x}+\frac{\sigma_{\mathrm{eff}}}{n_{\mathrm{ih}}} \frac{\partial p_{\mathrm{ih}}}{\partial x}=-\frac{\partial \phi}{\partial x}, \\
& \frac{\partial v_{\mathrm{il}}}{\partial t}+v_{\mathrm{il}} \frac{\partial v_{\mathrm{il}}}{\partial x}+Q_{\mathrm{un}} \frac{\sigma_{\mathrm{eff}}}{n_{\mathrm{il}}} \frac{\partial p_{\mathrm{il}}}{\partial x}=-Q_{\mathrm{un}} \frac{\partial \phi}{\partial x}, \\
& \frac{\partial p_{\mathrm{ih}}}{\partial t}+v_{\mathrm{ih}} \frac{\partial p_{\mathrm{ih}}}{\partial x}+3 p_{\mathrm{ih}} \frac{\partial v_{\mathrm{ih}}}{\partial x}=0, \\
& \frac{\partial p_{\mathrm{il}}}{\partial t}+v_{\mathrm{il}} \frac{\partial p_{\mathrm{il}}}{\partial x}+3 p_{\mathrm{il}} \frac{\partial v_{\mathrm{il}}}{\partial x}=0,
\end{aligned}
$$

with

$\sigma_{\text {eff }}=T_{\mathrm{i}} / T$ eff $=\rho_{\mathrm{h}} \sigma_{\mathrm{h}}+\rho_{\mathrm{l}} \sigma_{\mathrm{l}} ; \quad T \mathrm{i}=\rho_{\mathrm{h}} T_{\mathrm{ih}}+\rho_{\mathrm{l}} T_{\mathrm{il}} ;$

and $\quad \mathrm{T}_{\mathrm{eff}}=\frac{\mathrm{T}_{\mathrm{ec}} \mathrm{T}_{\mathrm{ew}}}{\mu \mathrm{T}_{\mathrm{ew}}+v \mathrm{~T}_{\mathrm{ec}}}$.

The corresponding Poisson's equation is

$\frac{\partial^{2} \phi}{\partial x^{2}}=n_{\mathrm{ec}}+n_{\mathrm{ew}}-n_{\mathrm{ih}}-n_{\mathrm{il}}$,

with $\mathrm{n}_{\mathrm{e}}=\mathrm{n}_{\mathrm{ec}}+\mathrm{n}_{\mathrm{ew}}=\mu \mathrm{e}^{\frac{\phi}{\mu+\nu \beta}}+v \mathrm{e}^{\frac{\beta \phi}{\mu+\nu \beta}}$.

The subscripts i, e, c, w, h, and 1 refer to ion, electron, cold and warm electrons and heavy and light mass ions respectively while $\sigma_{\mathrm{k}}\left(=T_{\mathrm{ik}} / T_{\mathrm{eff}} ; k=h, l\right), \beta\left(=T_{\mathrm{ec}} / T_{\mathrm{ew}}\right)$ and $Q_{\text {un }}\left(=m_{\mathrm{ih}} / m_{\mathrm{il}}\right)$ are the finite ion temperature effect, cold to hot electron temperature ratio and heavy to light ion mass ratio respectively and other notations have their usual meaning. All the number densities, viz $\mu, v, \rho_{\mathrm{h}}$ and $\rho_{1}$, which are the initial densities for cold and warm electrons and heavy and light ions respectively, are normalized to the equilibrium plasma density $n_{0}\left(\mu+v=\rho_{\mathrm{h}}+\rho_{\mathrm{l}}=1\right)$, the ion pressure $p_{\mathrm{ik}}(k=h, l)$ is normalized to ion equilibrium pressure $p_{0}\left(=n_{0} T_{\mathrm{i}}\right)$ and other variables, viz $\mathrm{t}$, $\mathrm{x}, \quad v_{\mathrm{i}}$ and $\phi$ are normalized to the reciprocal of ion plasma frequency $\omega_{\mathrm{pi}}^{-1}\left(\omega_{\mathrm{pi}}=\sqrt{\frac{4 \pi n_{0} e^{2}}{m_{\mathrm{ih}}}}\right)$, effective Debye length $\lambda_{\text {eff }}\left(=\sqrt{\frac{T_{\text {eff }}}{4 \pi n_{0} e^{2}}}\right)$, effective ion acoustic velocity $v_{\text {eff }}\left(=\sqrt{\frac{T_{\text {eff }}}{m_{\text {ih }}}}\right)$ and $\frac{T_{\text {eff }}}{e}$, respectively. The Mach number $M$

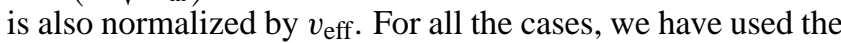
heavy ion mass as the normalization parameter.

We assume following normalized boundary conditions

at $|\mathrm{x}| \rightarrow \infty, \mathrm{v}_{\mathrm{ik}} \rightarrow \mathrm{v}_{0 \mathrm{k}}, \sum \mathrm{p}_{\mathrm{ik}} \rightarrow 1, \sum \mathrm{n}_{\mathrm{ik}} \rightarrow 1$

and $\phi \rightarrow 0$,

which also implies that

at $\quad|\mathrm{x}| \rightarrow \infty, \mathrm{p}_{\mathrm{ik}} \rightarrow \rho_{\mathrm{k}} \frac{\sigma_{\mathrm{k}}}{\sigma_{\mathrm{eff}}}$, and $\mathrm{n}_{\mathrm{ik}} \rightarrow \rho_{\mathrm{k}}$

Solving these fluid equations in a stationary wave frame (Ghosh et al., 1996; Ghosh and Iyengar, 2002), we have obtained the corresponding warm ion densities as

$$
\begin{aligned}
& n_{\mathrm{ih}}=\frac{\rho_{\mathrm{h}}}{2 \sqrt{3 \sigma_{\mathrm{h}}}}\left[\left\{\left(M+\sqrt{3 \sigma_{\mathrm{h}}}\right)^{2}-2 \phi\right\}^{1 / 2}\right. \\
& \left.-\left\{\left(M-\sqrt{3 \sigma_{\mathrm{h}}}\right)^{2}-2 \phi\right\}^{1 / 2}\right], \\
& n_{\mathrm{il}}=\frac{\rho_{\mathrm{l}}}{2 \sqrt{3 \sigma_{1}}}\left[\left\{\left(\frac{M}{\sqrt{Q_{\mathrm{un}}}}+\sqrt{3 \sigma_{\mathrm{l}}}\right)^{2}-2 \phi\right\}^{1 / 2}\right. \\
& \left.-\left\{\left(\frac{M}{\sqrt{Q_{\mathrm{un}}}}-\sqrt{3 \sigma_{1}}\right)^{2}-2 \phi\right\}^{1 / 2}\right] \text {. }
\end{aligned}
$$

Integrating Poisson's equation with these ion densities, we have obtained the required form of the Sagdeev pseudopotential as

$$
\begin{aligned}
& \psi_{\text {unmag }}(\phi)= \\
& -\left[(\mu+v \beta)\left\{\mu\left(e^{\frac{\phi}{\mu+\nu \beta}}-1\right)+\frac{v}{\beta}\left(e^{\frac{\beta \phi}{\mu+\nu \beta}}-1\right)\right\}\right. \\
& +\frac{\rho_{\mathrm{h}}}{6 \sqrt{3 \sigma_{\mathrm{h}}}}\left\{\left[\left(M+\sqrt{3 \sigma_{\mathrm{h}}}\right)^{2}-2 \phi\right]^{3 / 2}-\left(M+\sqrt{3 \sigma_{\mathrm{h}}}\right)^{3}\right. \\
& \left.-\left[\left(M-\sqrt{3 \sigma_{\mathrm{h}}}\right)^{2}-2 \phi\right]^{3 / 2}+\left(M-\sqrt{3 \sigma_{\mathrm{h}}}\right)^{3}\right\} \\
& +\frac{\rho_{\mathrm{l}}}{6 \sqrt{3 \sigma_{1}}}\left\{\left[\left(\frac{M}{\sqrt{Q_{\mathrm{un}}}}+\sqrt{3 \sigma_{1}}\right)^{2}-2 \phi\right]^{3 / 2}\right. \\
& -\left(\frac{M}{\sqrt{Q_{\mathrm{un}}}}+\sqrt{3 \sigma_{1}}\right)^{3}-\left[\left(\frac{M}{\sqrt{Q_{\mathrm{un}}}}-\sqrt{3 \sigma_{1}}\right)^{2}-2 \phi\right]^{3 / 2} \\
& \left.\left.+\left(\frac{M}{\sqrt{Q_{\mathrm{un}}}}-\sqrt{3 \sigma_{1}}\right)^{3}\right\}\right],
\end{aligned}
$$

which satisfies the following energy equation

$$
\frac{1}{2}\left(\frac{d \phi}{d \eta}\right)^{2}+\psi_{\text {unmag }}(\phi)=0,
$$

the subscript denoting the case of an unmagnetized plasma. For simplicity, the two species of ions are considered to have equal temperatures, viz, $\sigma=\sigma_{\mathrm{h}}=\sigma_{1}$ and their initial velocities $v_{0 \mathrm{k}}=0$. 


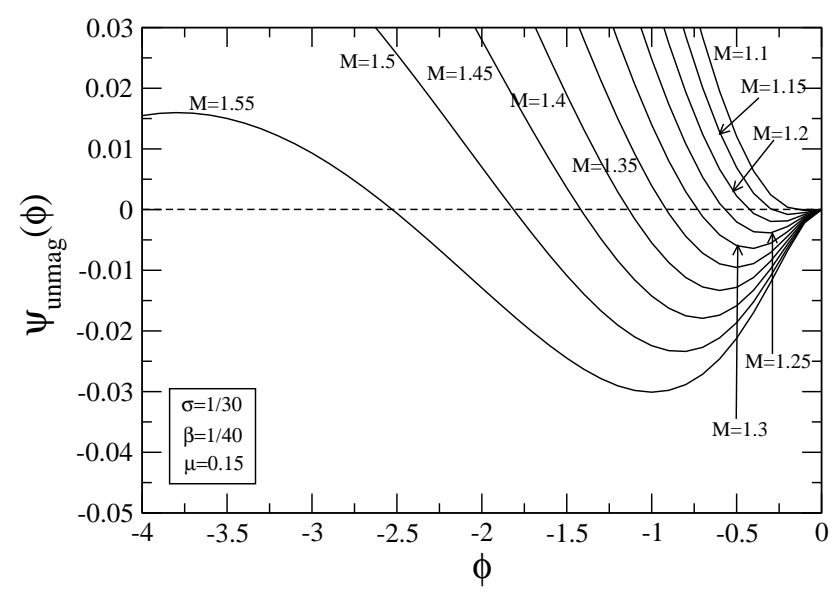

Fig. 1. Sagdeev pseudopotential curves for rarefactive ion acoustic solitary waves in an unmagnetized plasma for different Mach numbers; $M=1.1$ to 1.55 .

The solitary wave solutions are obtained from the Sagdeev pseudopotential by satisfying the following boundary conditions (Ghosh et al., 1996; Ghosh and Iyengar, 1997),

$\psi(\phi=0)=\left.\frac{\partial \psi(\phi)}{\partial \phi}\right|_{\phi=0}=0 ;\left.\quad \frac{\partial^{2} \psi(\phi)}{\partial \phi^{2}}\right|_{\phi=0}<0 ;$

$\psi\left(\phi_{0}\right)=0$

$\psi(\phi)<0$ for $0<|\phi|<\left|\phi_{0}\right|$.

These conditions on $\psi_{\text {unmag }}(\phi)$ also predict the limiting (critical) value of the Mach number $(M)$ for a solitary wave in an unmagnetized plasma. The existence of a solitary wave solution is, thus, implied by the following inequality

$\frac{\rho_{\mathrm{h}}}{M^{2}-3 \sigma_{\mathrm{h}}}+\frac{\rho_{\mathrm{l}}}{\frac{M^{2}}{Q_{\text {un }}}-3 \sigma_{\mathrm{l}}}<1$.

Equation (16) is the boundary condition for $M$. It also implies that the limiting value of $M$ depends on the parameters like ion mass ratio $\left(Q_{\mathrm{un}}\right)$, ion concentrations $\left(\rho_{\mathrm{h}, 1}\right)$ and ion temperatures $\left(\sigma_{\mathrm{k}}, k=h, l\right)$. Figure 1 shows the Sagdeev pseudopotential curves for a single ion $(Q=1)$ plasma for a chosen set of parameters, viz, $\beta=1 / 40, \mu=0.15$ and $\sigma=1 / 30$, respectively. The Mach number $M$ varies from $M=1.1$ to 1.55 . Details of the rarefactive ion acoustic solitary wave solutions in a multi-ion plasma have been discussed elsewhere (Ghosh and Iyengar, 2002). For the present work we are focusing to the anomalous width variation of the large amplitude solutions which we shall discuss in the next Section.

\subsection{Case II: Oblique propagation (magnetized plasma)}

In this case, the plasma is assumed to have a magnetic field parallel to $z$ direction and the wave is propagating obliquely in the $y-z$ plane with an angle $\theta$ with the ambient magnetic field. The parameter $\alpha$ denotes the ratio of the ion cyclotron and ion plasma frequencies $\left(\alpha=\omega_{\mathrm{ci}} / \omega_{\mathrm{pi}}\right)$. A parallel beam of $\mathrm{O}^{+}$ions has been considered moving with a beam velocity $u_{b}$. The bulk of the plasma is consisted of $\mathrm{H}^{+}$ions which are magnetized. Since the $\mathrm{O}^{+}$ions are much heavier, they are assumed to be unmagnetized. Following Ghosh and Iyengar (2002), we write the set of the normalized fluid equations as

$$
\begin{aligned}
\frac{\partial N_{\mathrm{b}}}{\partial t}+\nabla \cdot\left(N_{\mathrm{b}} \boldsymbol{v}_{\mathbf{b}}\right) & =0, \\
\frac{\partial \boldsymbol{v}_{\mathbf{b}}}{\partial t}+\left(\boldsymbol{v}_{\mathbf{b}} \cdot \nabla\right) \boldsymbol{v}_{\mathbf{b}}= & -Q_{\mathrm{mag}}\left[\boldsymbol{\nabla} \phi+3 \sigma N_{\mathrm{b}} \boldsymbol{\nabla} N_{\mathrm{b}}\right], \\
\frac{\partial N_{\mathrm{i}}}{\partial t}+\nabla \cdot\left(N_{\mathrm{i}} \boldsymbol{v}_{\mathbf{i}}\right)= & 0, \\
\frac{\partial \boldsymbol{v}_{\mathbf{i}}}{\partial t}+\left(\boldsymbol{v}_{\mathbf{i}} \cdot \nabla\right) v_{\mathrm{i}}= & -\left[\nabla \phi+3 \sigma N_{\mathrm{i}} \nabla N_{\mathrm{i}}\right] \\
& +\alpha\left(\boldsymbol{v}_{\mathbf{i}} \times \boldsymbol{b}\right),
\end{aligned}
$$

where $N_{\mathrm{i}, \mathrm{b}}=n_{\mathrm{i}, \mathrm{b}} / \rho_{\mathrm{i}, \mathrm{b}}$ respectively, $\rho_{\mathrm{i}, \mathrm{b}}$ being the ambient densities of $\mathrm{H}^{+}$ions and $\mathrm{O}^{+}$beam ions, respectively. The subscripts $i$ and $b$ denote $\mathrm{H}^{+}$ions and $\mathrm{O}^{+}$beam ions respectively. The electron density is given by Eq. (8). In the case of the magnetized plasma, since the bulk is assumed to be consisted of $\mathrm{H}^{+}$ions, the parameters are normalized by $\mathrm{H}^{+}$ion mass $\left(Q_{\text {mag }}=m_{\mathrm{i}} / m_{\mathrm{b}}\right)$. To evaluate Eqs. (17-20), we assume quasineutrality condition (Buti, 1980b). Since the ion Debye length is much smaller than the ion gyroradius $(\alpha \ll 1)$, that justifies our assumption (Sutradhar and Bujarbarua, 1988).

Assuming the massive ions as adiabatic $(\gamma=3)$ (Ghosh et al., 1996; Roychoudhury and Bhattacharyya, 1987), the equation of state becomes

$p_{\mathrm{i}, \mathrm{b}} \propto n_{\mathrm{i}, \mathrm{b}}^{3}$.

The charge neutrality condition implies that

$n_{\mathrm{i}}=n_{\mathrm{e}}-n_{\mathrm{b}}$.

The stationary state solution is obtained by assuming the transformation

$\eta=k_{\mathrm{y}} y+k_{\mathrm{z}} z-M t$,

where $M$ is the Mach no. and $k_{\mathrm{y}, \mathrm{z}}$ are the corresponding direction cosines for the oblique propagation.

Using Eq. (22), Eqs. (17-20) reduce to

$$
\begin{aligned}
& \frac{d}{d \eta}\left[\frac{1}{2}\right. \frac{1}{N_{\mathrm{i}}} \frac{d^{2}}{d \eta^{2}}\left\{\left(\frac{M}{N_{\mathrm{i}}}\right)^{2}+3 \sigma N_{\mathrm{i}}^{2}+2 \phi\right\} \\
&\left.+\alpha^{2}\left\{\frac{1}{N_{\mathrm{i}}}+\frac{k_{\mathrm{z}}^{2}}{M^{2}}\left(\sigma N_{\mathrm{i}}^{3}+N_{\mathrm{i}}^{\prime}\right)\right\}\right]=0,
\end{aligned}
$$

where

$N_{\mathrm{i}}^{\prime}=\int N_{\mathrm{i}} d \phi$.

Solving the coupled Eqs. (21) and (23) we get

$\frac{1}{2}\left(\frac{d \phi}{d \eta}\right)^{2}+\psi_{\mathrm{mag}}(\phi)=0$, 
where $\psi_{\text {mag }}(\phi)$ is the Sagdeev pseudopotential for a magnetized plasma.

$\psi_{\text {mag }}(\phi)=V(\phi) \frac{L(\phi)}{H(\phi)}$,

$V(\phi), L(\phi), H(\phi)$ being functions of $\phi, M$ and other parameters.

$$
\begin{aligned}
V(\phi) & =\alpha^{2} N_{\mathrm{i}}^{6}, \\
H(\phi) & =\left[N_{\mathrm{i}}^{3}+\left(3 \sigma N_{\mathrm{i}}^{4}-M^{2}\right) \frac{\partial N_{\mathrm{i}}}{\partial \phi}\right]^{2}, \\
L(\phi) & =\left[k_{\mathrm{y}}^{2} \phi+\frac{1}{2} M^{2}\left(\frac{s}{N_{\mathrm{i}}}\right)^{2}+(w-3 h)\right] \\
& +k_{\mathrm{z}}^{2}\left[h-\frac{1}{N_{\mathrm{i}}}\left(a+s^{\prime}\right)+\frac{1}{2}\left(\frac{w}{M}\right)^{2}\right],
\end{aligned}
$$

where

$$
s=1-N_{\mathrm{i}}, \quad t=\sigma\left(1-N_{\mathrm{i}}^{3}\right), \quad h=\frac{1}{2} \sigma\left(1-N_{\mathrm{i}}^{2}\right) ;
$$

$s^{\prime}=N_{\mathrm{i}}^{\prime}(\phi=0)-N_{\mathrm{i}}^{\prime}, \quad a=\sigma s, \quad w=t+s^{\prime} ;$

$N_{\mathrm{b}}=\frac{1}{2 \sqrt{3 \sigma}}\left[M_{\mathrm{b}+}\left(1-\frac{2 \phi}{M_{\mathrm{b}+}^{2}}\right)^{1 / 2}-M_{\mathrm{b}-}\left(1-\frac{2 \phi}{M_{\mathrm{b}-}^{2}}\right)^{1 / 2}\right]$

$M_{\mathrm{b} \pm}=\frac{1}{\sqrt{Q_{\mathrm{mag}}}}\left(M-k_{\mathrm{z}} u_{\mathrm{b}}\right) \pm \sqrt{3 \sigma}$.

To ascertain the existence of the solitary ion acoustic wave, the Sagdeev pseudopotential $\left(\psi_{\text {mag }}(\phi)\right)$ should satisfy the usual boundary conditions analogous to the unmagnetized case (Eq. (15)) (Ghosh et al., 1996). The inequality conditions thus obtained appeared to be more restricted for the magnetized plasma. These conditions are also found to depend on the specific parameter ranges. The inequality conditions for different parameter regions are listed below

\section{Case a)}

For,

$$
\frac{\rho_{\mathrm{i}}}{M^{2}-3 \sigma}+\frac{\rho_{\mathrm{b}}}{M_{\mathrm{b}}^{2}-3 \sigma}<1, \quad M>\sqrt{3 \sigma} \text {; }
$$

or,

$$
\begin{aligned}
& \frac{\rho_{\mathrm{i}}}{M^{2}-3 \sigma}+\frac{\rho_{\mathrm{b}}}{M_{\mathrm{b}}^{2}-3 \sigma}>1, \quad M<\sqrt{3 \sigma} ; \\
& \frac{\rho_{\mathrm{b}}\left(1-3 \sigma \frac{k_{\mathrm{z}}^{2}}{M^{2}}\right)}{M_{\mathrm{b}}^{2}-3 \sigma}>1-\frac{k_{\mathrm{z}}^{2}}{M^{2}}\left(\rho_{\mathrm{i}}+3 \sigma\right) .
\end{aligned}
$$

Case b)

For

$$
\frac{\rho_{\mathrm{i}}}{M^{2}-3 \sigma_{\mathrm{h}}}+\frac{\rho_{\mathrm{b}}}{M_{\mathrm{b}}^{2}-3 \sigma}>1, \quad M>\sqrt{3 \sigma} ;
$$

or,

$$
\frac{\rho_{\mathrm{i}}}{M^{2}-3 \sigma_{\mathrm{h}}}+\frac{\rho_{\mathrm{b}}}{M_{\mathrm{b}}^{2}-3 \sigma}<1, \quad M<\sqrt{3 \sigma} ;
$$

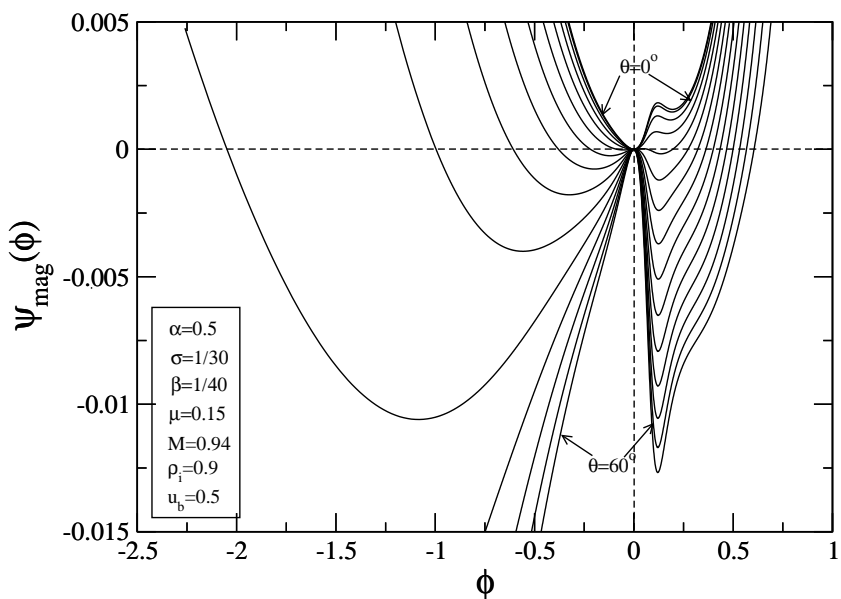

Fig. 2. Sagdeev pseudopotential curves for rarefactive ion acoustic solitary waves in a magnetized plasma for different angles of propagation; $\theta=0^{\circ}$ to $60^{\circ}$.

$$
\frac{\rho_{\mathrm{b}}\left(1-3 \sigma \frac{k_{\mathrm{z}}^{2}}{M^{2}}\right)}{M_{\mathrm{b}}^{2}-3 \sigma}<1-\frac{k_{\mathrm{z}}^{2}}{M^{2}}\left(\rho_{\mathrm{i}}+3 \sigma\right) .
$$

It is found that unlike the unmagnetized case, where the solutions are necessarily supersonic $(M>1)$, the solutions for a magnetized plasma generally fall in the subsonic range $(M<1)$. The solutions are best obtained for a fairly oblique propagation while for a parallel, or near-parallel propagation, the model based on an unmagnetized plasma (viz, Case 1) is more appropriate. A corresponding set of Sagdeev pseudopotential curves have been shown in Fig. 2 for different angles $\left(\theta=0^{\circ}\right.$ to $\left.60^{\circ}\right)$. The parameters are chosen to be $\beta=1 / 40, \quad \mu=0.15, \alpha=0.5$ and $\sigma=1 / 30$. The $\mathrm{H}^{+}$and $\mathrm{O}^{+}$ion densities are 0.9 and 0.1 , respectively and the normalized beam ion speed for $\mathrm{O}^{+}$ion is 0.5 . The solutions remain subsonic, the Mach number being $M=0.94$. It is observed that for a near-parallel propagation, the Sagdeev pseudopotential remains always positive discarding any solitary wave solution. This also implies that the present model is not really appropriate for a near-parallel propagation. On the other hand for a large $\theta$ the rarefactive solitary wave solutions may turn to be a weak double layer and for a larger obliqueness, the solution ceases to exist.

It is observed that the solutions for a magnetized plasma are more restricted compared to an unmagnetized plasma. Especially for those parameter ranges for which $H(\phi) \rightarrow 0$, the solutions cease to exist due to singularities in $\psi_{\text {mag }}(\phi)$. In those parameter regions, the Sagdeev pseudopotentials tend to be discontinuous and that restricts the corresponding solitary wave solutions even further. Any physical solution of the energy equation, viz, Eq. (24) ceases to exist near the vicinity of such parameter regions. The detail parametric analysis of the singularities of the Sagdeev pseudopotential for a magnetized plasma will be presented elsewhere.

Using Eq. (25) in the energy equation, viz, Eq. (24), and integrating it numerically, we have obtained the 


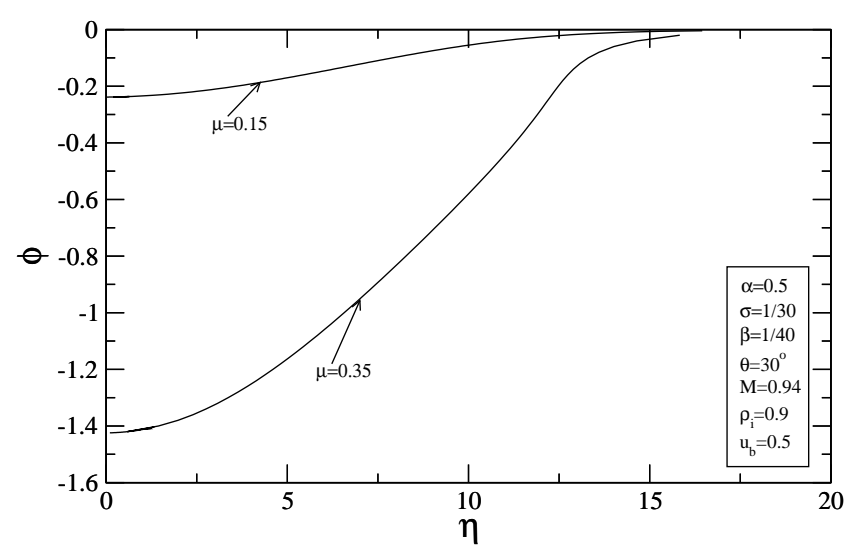

Fig. 3. Potential profiles for rarefactive ion acoustic solitary waves in a magnetized plasma for two different cold electron concentrations, viz $\mu=0.15$ and 0.35 .

corresponding rarefactive solitary wave solutions for a magnetized plasma. Figure 3 shows potential profiles of rarefactive ion acoustic solitary waves for two different initial cold electron concentrations, viz, $\mu=0.15$ and 0.35 , propagating obliquely with an angle $\theta=30^{\circ}$ with the ambient magnetic field. It shows that the amplitude increases considerably with increasing $\mu$. It also shows that the solution with larger amplitude also have a larger width (Fig. 3). It is this interesting property of a large amplitude rarefactive solitary wave which leads us to the following analysis of the width-amplitude variation profiles.

\section{Anomalous width}

Figure 3 readily shows that, with an increasing amplitude, the width of the rarefactive solitary wave may increase. In order to get a more complete scenario of the width-amplitude variation profile, we integrated the energy equations, viz, Eqs. (14) and (24), numerically. The measured half-width of the resulting potential profile has been plotted against the corresponding amplitude. Figure 4 shows the width-amplitude variation profile for an unmagnetized plasma for different electron temperature ratios $(\beta=1 / 40$ to $1 / 200)$. The plasma is chosen to have a single ion species with other parameters being the same as in Fig. 1. The shape of the width-amplitude variation profiles looks like an "asymmetric parabola", which readily shows two distinct regions of variations. For a sufficiently small negative amplitude (e.g. $\left|\phi_{0}\right|<0.5, \phi_{0}$ being the amplitude), the width decreases with the increasing amplitude (region I) while for a large amplitude solution $\left(\left|\phi_{0}\right|>0.5\right)$, the width starts to increase with the increasing amplitude (Region III) (Ghosh et al., 1996). Extensive parametric investigations have shown that the overall patterns of the width-amplitude variation profiles remain consistent for a wide range of parameters for a single ion (Ghosh and Iyengar, 1997) as well as multi-ion plasmas (Ghosh and Iyengar, 2002). In fact, it appears to be a basic characteristics of a

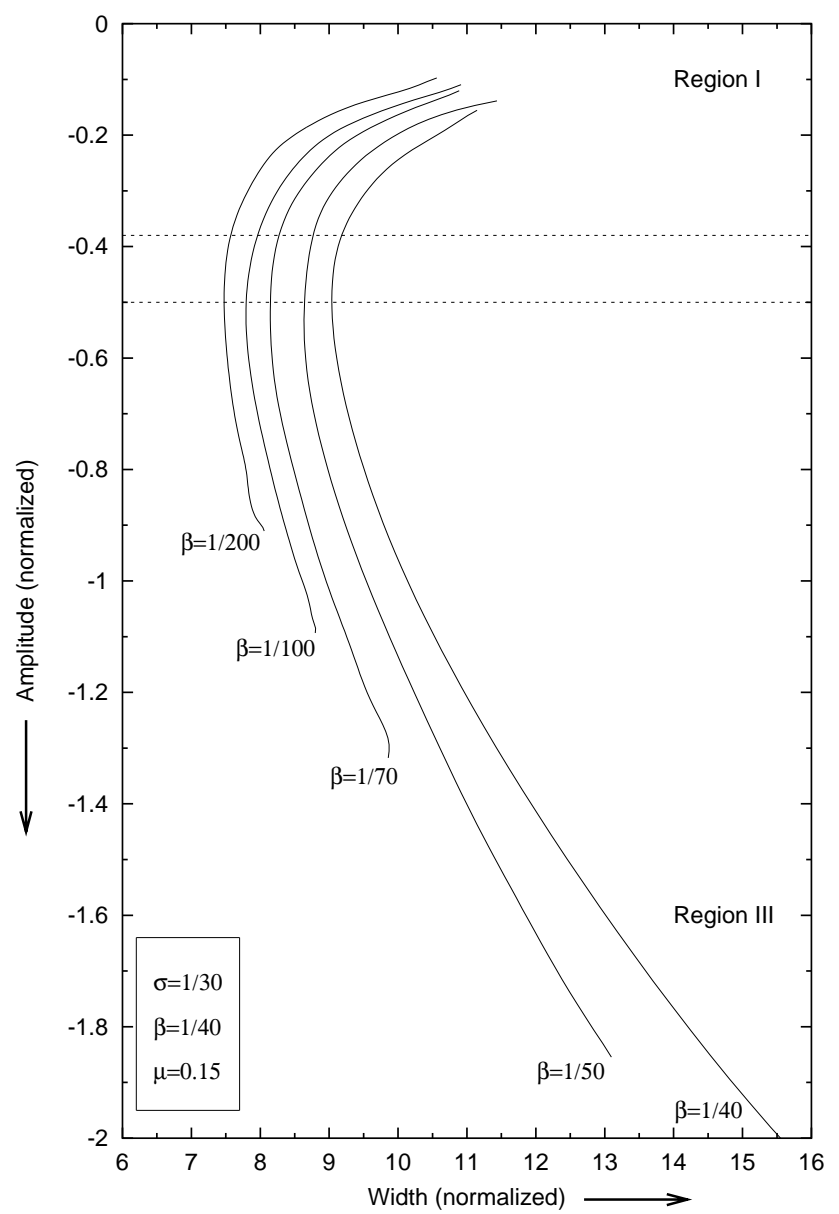

Fig. 4. Amplitude-width variation profiles $\left(\phi_{0} \mathrm{Vs} \delta\right)$ for a rarefactive ion acoustic solitary wave in an unmagnetized plasma for different $\beta$ values; $\beta=1 / 40$ to $1 / 200$ (single ion).

fully nonlinear solution of the rarefactive ion acoustic solitary wave. An elaborate exploration of the parameter space also established that the ambient densities of the different ion and electron species play crucial roles in determining the corresponding variation patterns. This model is found to be appropriate for a parallel or near-parallel propagation of the wave where the plasma can well be approximated as unmagnetized.

This analysis is further extended to an oblique propagation where the effect of the presence of the ambient magnetic field has also been taken into account. In order to obtain the corresponding rarefactive ion acoustic solitary wave solution, we have solved the coupled energy equations for the magnetized plasma, viz, Eqs. (24) and (25), numerically. We have chosen a propagation angle of $40^{\circ}$ with the ambient magnetic field while the other parameters remain the same as those in Fig. 2. The width-amplitude variation profiles are obtained for different magnetic fields, $\alpha$ ranging from 0.2 to 0.55 . Figure 5 shows the corresponding width-amplitude variation profiles for different values of $\alpha$. It shows that a similar trend is also observed for a magnetized plasma. For smaller amplitudes (e.g. $\left|\phi_{0}\right|<0.3$ ) the width decreases with the increasing 


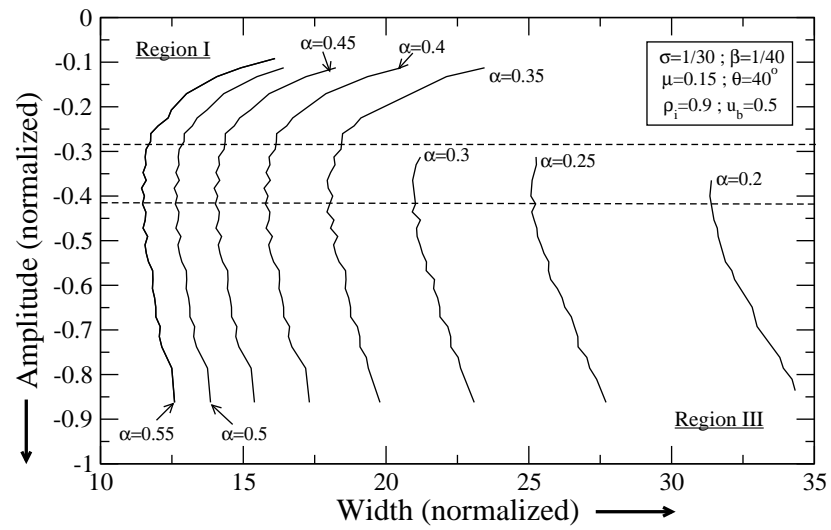

Fig. 5. Amplitude-width variation profiles $\left(\phi_{0} \mathrm{Vs} \delta\right)$ for a rarefactive ion acoustic solitary wave in a magnetized plasma for different magnetic fields; $\alpha=0.2$ to 0.55 .

amplitude while for a larger amplitude it shows just an opposite trend. This very typical trend of the width-amplitude variation pattern has motivated us to compare our analytical results with the recent space plasma observations.

The discrepancies between the weakly nonlinear theory (Washimi and Taniuti, 1966) and the observations of the auroral acceleration region (Böstrom et al., 1989) was known for a long time. However, not many attempts were taken to cover the particular lacuna in the width-amplitude variation pattern. Previous theoretical analysis have suggested both the presence of ion acoustic modes (Lotko, 1983), or BGK ion holes in the phase space (Mäikii and Koskinen, 1989). It was considered that an ion acoustic mode necessarily show a decrease in the width with increasing amplitude while an opposite trend indicates the presence of BGK modes in the space. However, unlike electron solitary holes (Muschietti et al., 1999b; Maslov and Schamel, 1993), the observed rarefactive structures in the auroral region (Temerin et al., 1982; Böstrom et al., 1989) were mainly interpreted as the evolution of ion acoustic mode (Reddy and Lakhina, 1991). Berthomier et al. (1998) have shown that a fully nonlinear rarefactive ion acoustic wave in an unmagnetized plasma may show an increase in the width with increasing amplitude. Previous satellite observations showed an apparently "random" variation of the width with amplitude where no regular correlation could be observed but recent satellites are more capable with higher data rates and high resolution particle detectors which have opened a wide opportunity to analyze different nonlinear structures. Significant works have been done by Cattell et al. (2001); Crumley et al. (2001) and Dombeck et al. (2001) in the auroral region on the basis of POLAR data. A particularly interesting finding was the width-amplitude variation of the low altitude rarefactive ion structures in the auroral region (Dombeck et al., 2001). It shows a clear trend of increasing width with increasing amplitude. Figure 6 shows one of the three EFI bursts as studied and presented by Dombeck et al. (2001). Though the

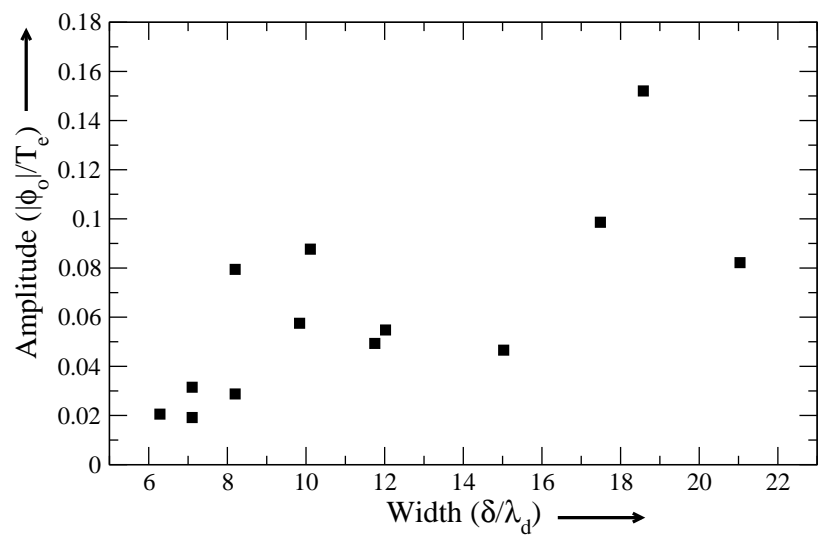

Fig. 6. POLAR observations of ion mode solitary potential structures by Dombeck et al. (2001); the normalized amplitude is plotted against the corresponding spatial width. The figure is reproduced from Dombeck et al., 2001.

variations do not show any clear pattern, the overall trend of increasing width with increasing amplitude seems to be quite prominent. Interestingly, for a few occasions, the variation pattern appears to be "double-valued", i.e., exactly same widths have been measured for two different amplitudes, one is considerably small while the other is much larger. This type of behavior seems to be consistent to our analytical results which shows a "parabolic" type of variation pattern. These apparent similarities lead us to a more quantitative comparison of our analytical results with the observed data.

In Dombeck et al. (2001), it was shown that the scale length of the observed structures are of the order of 10 to 50 Debye lengths which is consistent with our analytical results. However, the observed normalized amplitudes appear to be much smaller than our predicted values. A comparison between Figs. 4 and 5 further indicates that in case of an unmagnetized plasma, the theoretical estimation of the amplitude tends to be even larger, being further away from the observed values. One major source of discrepancies lie in the normalizing parameters. Our model is based on two electron temperatures whereas there is only a single measured electron temperature available in the data. Since the widthamplitude variation profiles are quite sensitive to the electron temperatures and concentrations (Ghosh and Iyengar, 1997), these may easily lead to a significant quantitative discrepancy. On the other hand, all theoretical analyses have shown that the presence of a second electron population (or negative ions) is necessary for the existence of a rarefactive ion acoustic solitary wave (Buti, 1980a). In the present case, we have chosen a magnetized plasma for the sake of our quantitative comparisons since it gives a more realistic estimation for the amplitude, e.g. for $\beta=1 / 40$ and $\delta \approx 14,\left|\phi_{0_{\text {mag }}}\right| \approx 0.9$ while $\left|\phi_{0_{\text {unmag }}}\right| \approx 1.8, \delta$ being the normalized width (Figs. 4 and 5). We have evaluated the width-amplitude variation profile for different $\beta$ values and compared them with the POLAR observations presented by Dombeck et al. (2001). We have chosen an appropriate parameter space and converted 
Table 1. Normalization parameters for observational and analytical results.

\begin{tabular}{cll}
\hline Parameters & Observational & Analytical \\
\hline$T_{\mathrm{e}}, T_{\mathrm{eff}}$ & $400 \mathrm{eV}$ & $58.39 \mathrm{eV}$ \\
& & $($ for $\beta=1 / 40)$ \\
$T_{\mathrm{ew}}$ & - & $400 \mathrm{eV}$ \\
$n_{0}$ & $0.5 \mathrm{~cm}^{-3}$ & $0.12 \mathrm{~cm}^{-3}$ \\
$\mu$ & - & 0.15 (normalized) \\
$v$ & - & 0.85 (normalized) \\
$u_{\mathrm{b}}$ & 0.5 (approx.) & 0.2 (normalized) \\
$\alpha$ & - & 0.5 \\
\hline
\end{tabular}

the parameters into their absolute (non-normalized) values. Figure 7 compares our analytical results (solid curves) to the observations of Dombeck et al. (2001). The points in the figure represent the observational data. Each curve presents a different set of normalization parameters corresponding to the particular $\beta$ value and the absolute values for the width and the amplitude displayed in the figure have been calculated accordingly. A set of parameters chosen for the comparison is listed in the Table 1. According to the data presented by Dombeck et al. (2001), the $\mathrm{H}^{+}$sound speed is around $200 \mathrm{~km} / \mathrm{s}$ while the $\mathrm{O}^{+}$beam speed is of the order of $100 \mathrm{~km} / \mathrm{s}$ which leads to a normalized beam speed of 0.5 .

Figure 7 shows that there is good qualitative agreement between the analytical results and observational data. It also appears that a model based on the assumption of oblique propagation (magnetized plasma) is more appropriate for interpreting the observed structures. Though the data presented by Dombeck et al. (2001) do not show any definite pattern at first sight, it appears from our analysis presented in Fig. 7 that a range of parameters (e.g. the varying $\beta$ values for the present case) would, perhaps, be more appropriate to reveal the underlying correlations between the width and the amplitude rather than a single set of parameters. It also indicates that with a more accurate and elaborate data set, the overall pattern of the width-amplitude variation will become clearer. However, a major source of ambiguity lies in the measurements of electronic parameters, viz, the temperatures and concentrations of different electron species which makes the quantitative agreement difficult. A kinetic, or semi-kinetic model may thus become helpful to bridge the gap and to offer a more complete theory of the overall width-amplitude variation profile for a rarefactive ion acoustic solitary wave. Nevertheless, the overall trend indicates good agreement with POLAR observations.

\section{Conclusion}

The present model describes the anomalous width variation for large amplitude ion holes observed in auroral plasma. A fully nonlinear solution for rarefactive ion acoustic solitary

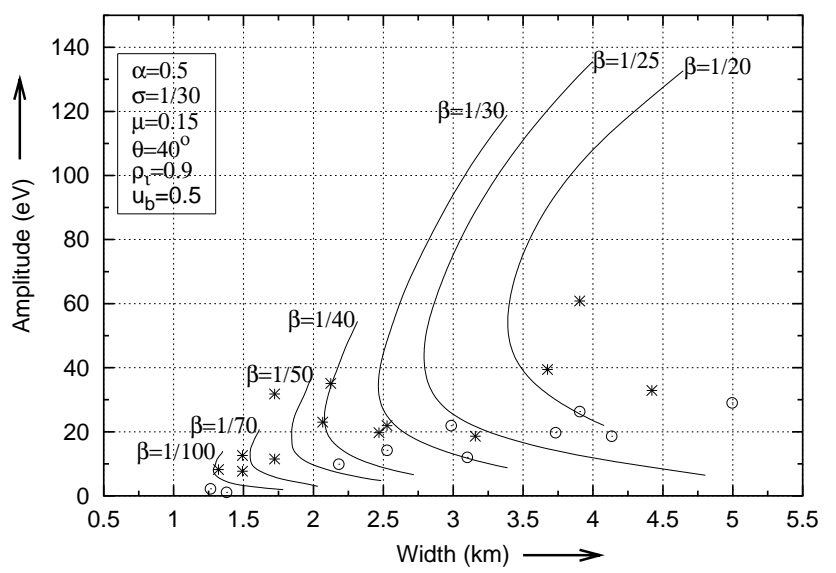

Fig. 7. Analytical width-amplitude profiles as compared with POLAR observations. Legends denote observational data for two EFI bursts as presented in Dombeck et al. (2001) while analytical curves (solid lines) are obtained for different cold to hot electron temperature ratios.

waves have been obtained for both unmagnetized and magnetized plasmas by using Sagdeev pseudopotential technique. For the case of a magnetized plasma, the charge neutrality condition is assumed to be valid. The analytical results show a very consistent width-amplitude variation pattern indicating an increase in the width with the increasing amplitude (anomalous width variation) for large amplitude solutions. Interestingly enough, recent POLAR observations also show a similar trend. From the data of the POLAR observations, Dombeck et al. (2001) have shown that the width of the rarefactive ion solitary waves in the auroral region tends to increase with the increasing amplitude. The analytical results obtained in our case have been compared to the observations of Dombeck et al. (2001) which shows a good qualitative agreement. However, a proper quantitative comparison needs more adequate information regarding the temperatures and concentrations of different species of ions and electrons present in the auroral region. The analytical results show that the width-amplitude variation profile is particularly sensitive to the different electron temperatures and concentrations. Theoretical models also suggest the presence of two electron species of different temperatures whereas most of the observational data presents a single electron temperature. As indicated by Berthomier et al. (1998), an appropriate quantitative agreement between the observations and the analytical results appears to be far too restricted in the parameter space.

In our theoretical model both the unmagnetized and magnetized plasmas are considered. The model based on the magnetized plasma is applicable for an oblique propagation while the model based on unmagnetized plasma will be more appropriate for a parallel or nearly parallel ( $\operatorname{small} \theta$ ) propagation. The analytical results show that the ion acoustic solitary wave solutions are more restricted for a magnetized plasma compared to an unmagnetized one. For the former case there exists point of singularities where, for a specific parameter 
set, the Sagdeev pseudopotential may blow up, thus restricting the existence domain of the solitary waves even further. The effect of the ambient magnetic field also appears to diminish the amplitude of the solitary wave significantly. This suggests that a theory based on the assumption of oblique propagation (magnetized plasma) is more appropriate for a realistic situation. Our model also shows that, for the chosen parameter regions, the ion acoustic solitary wave in a magnetized plasma propagates with a fairly large propagation angle, $\theta$ being greater than $30^{\circ}$. This theoretical estimation for the propagation angle of the solitary wave needs to be verified with recent CLUSTER data and will be communicated elsewhere.

In the present analysis we have focused our discussions to the width-amplitude variations of a rarefactive ion acoustic solitary wave. As we have compared our theoretical results with POLAR data presented by Dombeck et al. (2001), it shows that the theoretically estimated amplitudes of the solitary wave in our model remain much larger than that observed in auroral plasma. We have already discussed that one major source of such discrepancies lie in the measurement of the normalization parameters (viz, $\mu, v$ and $T_{\text {eff }}$ ). However, in the real low-altitude magnetosphere, there exist other complex physical processes involving the impact of ionospheric boundaries and possible wave-wave couplings between ion acoustic and ion cyclotron or Alfven waves which may also affect the formation of solitary waves in the auroral region. Such analyses are beyond the scope of the present work and a further generalization of the model may provide a better agreement of the theoretical estimation with the satellite observations.

So far the study of the overall width-amplitude variation profile has received scant attention. The present simple model shows a consistent variation pattern for all the parameter ranges which is also supported by the recent observations in auroral plasma. This indicates that a more complete theory for a fully nonlinear solution of large amplitude solitary waves is needed to describe the auroral observations more appropriately. A more rigorous verification of both the analytical results and the observational data may help to understand the underlying physical processes involving in the change of the variation pattern from Region I (decreasing width) to Region III (increasing width) while it will also improve the current theoretical model for more realistic situations in low-altitude magnetosphere. A more complete analysis of the fully nonlinear solutions of the ion acoustic solitary waves will be communicated elsewhere.

Acknowledgements. Part of the work has been carried out in Radio Science Center for Space and Atmosphere, Kyoto University, Uji, Kyoto, Japan. One of the authors, S. S. Ghosh, thanks Y. Omura, Radio Science Center for Space and Atmosphere, Kyoto, Japan for his kind help and the facilities provided by him. The authors thank their colleagues in Indian Institute of Geomagnetism, Navi Mumbai, India for continuing support and helpful discussion.

Edited by: A. S. Sharma

Reviewed by: two referees

\section{References}

Bale, S., Kellogg, P., Larson, D., Lin, R., Goetz, K., and Lepping, R.: Bipolar electrostatic structures in the shock transition region: Evidence of electron phase space holes, Geophys. Res. Lett., 25 , 2929-2932, 1998.

Barnes, C., Hudson, M. K., and Lotko, W.: Weak double layers in ion acoustic turbulence, Phys. Fluids, 28, 1055-1062, 1985.

Berthomier, M., Pottelette, R., and Malingre, M.: Solitary waves and weak double layers in a two-electron temperature auroral plasmas, J. Geophys. Res., 103, 4261-4270, 1998.

Böstrom, R., Gustafsson, G., Holback, B., Holmgren, G., Koshkinen, H., and Kintner, P.: Characteristics of solitary waves and double layers in the magnetospheric plasma, Phys. Rev. Lett., 61, 82-85, 1988.

Böstrom, R., Holback, B., and Holmgren, G.: Solitary structures in the magnetospheric plasma observed by Viking, Phys. Scr., 39, 782-786, 1989.

Bounds, S. R., Pfaff, R. F., Knowlton, S. F., Mozer, F. S., Temerin, M. A., and Kletzing, C. A.: Solitary potential structures associated with ion and electron beams near 1 Re altitudes, J. Geophys. Res., 104, 28,709-28,717, 1999.

Buti, B.: Ion acoustic holes in a two temperature plasma, Phys. Lett., 76A, 251-254, 1980.

Buti, B.: Nonlinear electron-acoustic wave in a multi-species plasma, J. Plasma Phys., 24, 169-180, 1980.

Cairns, R. A., Mamun, A. A., Bingham, R., Böstrom, R., Dendy, R. O., Nairn, C. M. C., and Shukla, P. K.: Electrostatic solitary structures in non-thermal plasmas, Geophys. Res. Lett., 22, 2709-2712, 1995.

Cattell, C. A., Dombeck, J., Wygnant, J. R., Hudson, M. K., Mozer, F. S., Temerin, M. A., Peterson, W. K., Kletzing, C. A., Russell, C. T., and Pfaff, R. F.: Comparisons of Polar satellite observations of solitary wave velocities in the plasma sheet boundary and the high altitude cusp to those in the auroral zone, Geophys. Res. Lett., 26, 425-428, 1999.

Cattell, C. A., Crumley, J., Dombeck, J., Lysak, R., Kletzing, C., Peterson, W. K., and Collin, C.: Polar observations of solitary waves at high and low altitudes and comparison to theory, Adv. Space Res., 28, 1631-1641, 2001.

Chanteur, G., Adam, J. C., Pellat, R., and Volokhitin, A. S.: Formation of ion acoustic double layers, Phys. Fluids, 26, 1584-1586, 1983.

Crumley, J. P., Cattell, C. A., Lysak, R. L., and Dombeck, J. P.: Studies of ion solitary waves using simulations including hydrogen and oxygen beams, J. Geophys. Res., 106, 6007-6015, 2001.

Dombeck, J., Cattell, C., Crumley, J., Peterson, W. K., Collin, H. L., and Kletzing, C.: Observed trends in auroral zone ion mode solitary wave structure characteristics using data from Polar, J. Geophys. Res., 106, 19013-19021, 2001.

Donver, P. O., Erikson, A. I., Böstrom, R., and Holback, B.: Freja multipole observations of electrostatic solitary structures, Geophys. Res. Lett., 21, 1827-1830, 1994.

Dubouloz, N., Pottelette, R., Malingre, M., and Treumann, R. A.: Generation of broadband electrostatic noise by electron acoustic solitons, Geophys. Res. Lett., 18, 155-158, 1991.

Dupree, T. H.: Theory of phase space density holes, Phys. Fluids, 25, 277-289, 1982.

Ergun, R. E., Carlson, C. W., McFadden, J. P., Mozer, F. S., Delory, G. T., Peria, W., Chaston, C. C., Temerin, M., Roth, I., Muschietti, L., Elphic, R., Strangeway, R., Pfaff, R., Cattell, C. A., Klumpar, D., Shelley, E., Peterson, W., Moebius, E., 
and Kistler, L.: FAST satellite observations of large amplitude solitary structures, Geophys. Res. Lett., 25, 2041-2044, 1998.

Franz, J., Kintner, P., and Pickett, J.: Polar observations of coherent electric field structures, Geophys. Res. Lett., 25, 1277-1280, 1998.

Ghosh, S. S., Ghosh, K. K., and Iyengar, A. N. S.: Large Mach number ion acoustic rarefactive solitary waves for a two electron temperature warm ion plasma, Physics of Plasmas, 4, 39393946, 1996.

Ghosh, S. S. and Iyengar, A. N. S.: Anomalous width variations for ion acoustic rarefactive solitary waves in a warm ion plasma with two electron temperatures, Physics of Plasmas, 5, 3204, 1997.

Ghosh, S. S. and Iyengar, A. N. S.: Anomalous width variation for rarefactive ion acoustic solitary waves in the presence of warm multiions, J. Plasma Phys., 67, 223-233, 2002.

Gray, P. C., Hudson, M. K., Lotko, W., and Bergmann, R.: Decay of ion beam driven acoustic waves into ion holes, Geophys. Res. Lett., 18, 1675-1678, 1991.

Hudson, M. K., Lotko, W., Roth, I., and Witt, E.: Solitary waves and double layers on auroral field lines, J. Geophys. Res., 88, 916-926, 1983.

Koskinen, H. E. J., Lundin, R., and Holback, B.: On the plasma environment of solitary waves and weak double layers, J. Geophys. Res., 95, 5921-5929, 1990.

Lotko, W.: Reflection dissipation of an ion acoustic soliton, Phys. Fluids, 26, 1771-1779, 1983.

Lotko, W.: Diffusive accelaration of auroral primaries, J. Geophys. Res., 91, 191-203, 1986.

Lotko, W. and Kennel, C. F.: Spiky ion acoustic waves in collisionless auroral plasma, J. Geophys. Res., 88, 381-394, 1983.

Mäikii, A. and Koskinen, H.: On theories attempting to explain observations of solitary waves and weak double layers in the auroral magnetosphere, Phys. Scr., 39, 787-793, 1989

Mäikii, A., Eriksson, A. I., Donver, P. O., Böstrom, R., Holback, B., Holmgren, G., and Koskinen, H. E. J.: A statistical survey of auroral solitary waves and weak double layers: 1 Occurrence and net voltage, J. Geophys. Res., 98, 15 521-15 530, 1993.

Mangeney, A., Salem, C., Lacombe, C., Bougeret, J. L., Perche, C., Manning, R., Kellogg, P. J., Goetz, K., Monson, S. J., and Bosqued, J. M.: WIND observations of coherent electrostatic waves in the solar wind, Ann. Geophys., 17, 307-320, 1999.

Marchenko, V. A. and Hudson, M. K.: Beam driven acoustic solitary waves in the auroral acceleration region, J. Geophys. Res., 100, 19791-19803, 1995.

Maslov, V. and Schamel, H.: Growing electron holes in drifting plasma, Phys. Lett., 78, 171-174, 1993.

Matsumoto, H. H., Kojima, H., Miyatake, T., Omura, Y., Okada, M., Nagano, I., and Tsutsui, M.: Electrostatic solitary waves (ESW) in the magnetotail: BEN waveforms observed by Geotail, Geophys. Res. Lett., 21, 2915-2918, 1994.
McFadden, J. P., Carlson, C. W., and Ergun, R. E.: Microstructure of the auroral acceleration region as observed by FAST, J. Geophys. Res., 104, 14,453-14 480, 1999.

Mozer, F. S., Ergun, R., Temerin, M., Cattell, C., Dombeck, J., and Wygant, J.: New features of time domain electric field structures in the auroral acceleration region, Phys. Rev. Lett., 79, 12811284, 1997.

Muschietti, L., Ergun, R. E., Roth, I., and Carlson, C. W.: Phase space electron holes along magnetic field lines, Geophys. Res. Lett., 26, 1093-1096, 1999a.

Muschietti, L., Ergun, R. E., Roth, I., and Carlson, C. W.: Phase space electron holes along magnetic field lines, Geophys. Res. Lett., 26, 1093-1096, 1999b.

Muschietti, L., Roth, I., Carlson, C. W., and Berthomier, M.: Modeling stretched solitary waves along magnetic field lines, Nonlin. Proc. Geophys., 9, 101-109, 2002.

Nishihara, K. and Tajiri, M.: Rarefaction ion acoustic solitons in two-electron-temperature plasma, J. Phys. Soc. Jpn., 50, 40474053, 1981.

Qian, S., Lotko, W., Hudson, M. K.: Dynamics of localized ionacoustic waves in a magnetized plasma, Phys. Fluids, 31, 13391346, 1988.

Qian, S., Lotko, W., Hudson, M. K.: Oxygen acoustic solitary waves in a magnetized plasma, J. Geophys. Res., 94, 1339-1346, 1989.

Reddy, R. V. and Lakhina, G. S.: Ion acoustic double layers and solitons in auroral plasma, Planet Space Sci., 39, 1343-1350, 1991.

Reddy, R. V., Lakhina, G. S., and Verheest, F.: Ion-acoustic double layers and solitons in multispecies auroral beam-plasma, Planet Space Sci., 39, 1055-1062, 1992.

Roychoudhury, R. K. and Bhattacharyya, S.: Effect of ion temperature on ion acoustic solitary waves: a pseudopotential appropach, Can. J. Phys., 65, 699-702, 1987.

Sato, T. and Okuda, H.: Ion acoustic double layers, Phys. Rev. Lett., 44, 740-743, 1980.

Schamel, H.: Kinetic theory of phase space vortices and double layers, Phys. Scr., T2/1, 328-337, 1982.

Sutradhar, S. and Bujarbarua, S.: Electron acoustic double layer in a current carrying magnetized plasma, Planet Space Sci., 36, 1009-1013, 1988.

Temerin, M., Cerny, K., Lotko, W., and Mozer, F. S.: Observations of solitary waves and double layers in the auroral plasma, Phys. Rev. Lett., 48, 1175-1179, 1982.

Turikov, V. A.: Electron phase space holes as localized BGK solutions, Phys. Scr., 30, 73-77, 1984.

Washimi, H. and Taniuti, T.: Propagation of ion acoustic solitary waves of small amplitude, Phys. Rev. Lett., 17, 996-998, 1966.

Yadav, L. L. and Sharma, S. R.: Obliquely propagating ion-acoustic solitons in a warm-ion magnetized plasma, Phys. Lett., 150A, 397-401, 1990. 